\title{
Sepsis por Lactobacillus reuteri en un recién nacido pretérmino: reporte de un caso
}

\author{
Sepsis due to Lactobacillus reuteri in an extreme preterm newborn: case report
}

\author{
Prof. Dra. Luz A. Celis Castañeda ${ }^{a}$, Dr. William J. Morales Camacho y Dr. Nelson M. Durán Ochoac
}

\begin{abstract}
RESUMEN
La utilización de probióticos en la población neonatal ha contribuido a una disminución de la morbilidad y mortalidad en esta población a través de la reducción en la incidencia de entidades como enterocolitis necrosante, infecciones asociadas al cuidado de la salud y sepsis neonatal. A pesar de los múltiples efectos beneficiosos descritos, este tipo de medicamentos debe ser administrado con precaución en los pacientes de alto riesgo, debido a que se han documentado reportes de casos de sepsis neonatal por Lactobacillus rhamnosus, Saccharomyces boulardii y Bifidobacterium, entre otros. Se presenta un caso atípico de sepsis neonatal por Lactobacillus reuteri en un recién nacido pretérmino extremo en una institución de alta complejidad en la ciudad de Bogotá D. C., Colombia. El desenlace, en nuestro caso, fue fatal, pese al manejo médico establecido.

Palabras clave: Lactobacillus reuteri, probióticos, sepsis neonatal.
\end{abstract}

\begin{abstract}
The use of probiotics in the neonatal population has contributed to a decrease in morbidity and mortality in this population through the reduction in the incidence of entities such as necrotizing enterocolitis, infections associated with health care and neonatal sepsis. Despite the multiple beneficial effects described due to the use of probiotics, this type of medication should be administered with caution in high-risk patients because reports of cases of neonatal sepsis by Lactobacillus rhamnosus, Saccharomyces boulardii and Bifidobacterium, among others, have been documented. We present an atypical case of neonatal sepsis due to Lactobacillus reuteri in an extreme preterm newborn in a highly complex institution in the city of Bogotá D. C., Colombia. The outcome in our case was fatal despite the established medical management.

Key words: Lactobacillus reuteri, probiotics, neonatal sepsis.
\end{abstract}

http: / / dx.doi.org/10.5546/ aap.2019.e509

a. Unidad de Neonatología, Hospital Simón Bolívar; Universidad El Bosque (UEB), Bogotá D. C., Colombia.

b. Pediatría, Universidad El Bosque (UEB), Bogotá D. C., Colombia.

c. Pediatría, Universidad de la Sabana, Bogotá D. C., Colombia.

Correspondencia:

Dr. William J. Morales Camacho: wmorales31@unab.edu.co

Financiamiento: Ninguno.

Conflicto de intereses: Ninguno que declarar.

Recibido: $12-8-2018$

Aceptado: $14-4-2019$
Cómo citar: Celis Castañeda LA, Morales Camacho WJ, Durán Ochoa NM. Sepsis por Lactobacillus reuteri en un recién nacido pretérmino: reporte de un caso. Arch Argent Pediatr 2019;117(5):e509-e513.

\section{INTRODUCCIÓN}

La utilización de probióticos en la población neonatal ha contribuido a una disminución significativa de la morbilidad y mortalidad en esta población a través de la reducción en la incidencia de entidades como enterocolitis necrosante, infecciones asociadas al cuidado de la salud y sepsis neonatal. ${ }^{1,2}$ Dentro de los efectos benéficos atribuidos a la utilización del Lactobacillus reuteri, se describe la mejor regulación en la expresión de citoquinas pro- y antiinflamatorias, que favorece un mejor funcionamiento de la microbiota intestinal, de la disminución de la pérdida de bacterias comensales y del riesgo de translocación bacteriana. ${ }^{1,2}$

A pesar de los múltiples efectos beneficiosos asociados a la utilización de probióticos, se han documentado reportes de casos de sepsis neonatal por Lactobacillus rhamnosus, Saccharomyces boulardii y Bifidobacterium, entre otros. ${ }^{3}$ Se reporta un caso de sepsis neonatal por Lactobacillus reuteri en un recién nacido pretérmino (RNPT) extremo en una institución de alta complejidad en la ciudad de Bogotá D. C., Colombia.

\section{CASO CLÍNICO}

Recién nacido pretérmino extremo de 27 semanas de edad gestacional por ultrasonografía temprana, embarazo controlado, perfil infeccioso negativo, parto por cesárea por preeclampsia grave, con administración de corticoide antenatal. Adaptación neonatal espontánea, Apgar 8-9-9, gases de cordón umbilical con $\mathrm{pH}$ : 7,24; exceso de base: $-4,6$, no sugestivos de asfixia perinatal. Peso al nacer: 840 gramos; talla: $35 \mathrm{~cm}$.

Después del nacimiento, desarrolló signos de dificultad respiratoria dados por tiraje subcostal e intercostal, por lo que se inició el soporte ventilatorio con pieza en T. Se trasladó 
a la Unidad de Cuidado Intensivo Neonatal. Por protocolo institucional, se administró una dosis de Lactobacillus reuteri (5 gotas/día) al ingresar a la Unidad. Se solicitaron hemocultivos luego de la administración del probiótico y se inició el manejo antibiótico con ampicilina y aminoglucósido. Se colocó un catéter umbilical arterial y venoso sin complicaciones. A las dos horas de vida, se realizó la intubación orotraqueal por el aumento de requerimientos de fracción inspirada de oxígeno $\left(\mathrm{FiO}_{2}\right)$, por lo que se inició la ventilación mecánica. Se administró 1 dosis de surfactante, con buena respuesta al tratamiento, que permitió el descenso de los parámetros ventilatorios, con radiografía de tórax dentro de los límites normales y sin datos de enfermedad de la membrana hialina. Se inició el estímulo trófico enteral con fórmula láctea por no disponer de un banco de leche en la Institución.

Durante la evolución clínica, se cambió a modo asistido controlado con volumen garantizado, con adecuada tolerancia ventilatoria. Paraclínicos, iniciales a las 6 horas de vida con evidencia de trombocitopenia leve, hipofosfatemia e hipermagnesemia (Tabla 1). A las 36 horas de vida, se realizó la extubación programada y se pasó a presión positiva continua en la vía aérea (continuous positive airway pressure; CPAP, por sus siglas en inglés) nasal modo control de presión-ventilación obligatoria continua (pressure control-continuous mandatory ventilation; PC$\mathrm{CMV}$, por sus siglas en inglés), con un episodio posterior de apnea, por lo que se inició cafeína y se aumentaron los parámetros ventilatorios.

En su segundo día de vida, se observó el deterioro progresivo a nivel hemodinámico y respiratorio, con el desarrollo de distermias y la persistencia de episodios de apneas hasta requerir una nueva intubación. Se obtuvo el reporte preliminar de hemocultivos a las 30 horas con crecimiento de cocos Gram-positivos, por lo que se reajustó la dosis de ampicilina en el contexto de un recién nacido con alta probabilidad de sepsis temprana. Debido a su inestabilidad hemodinámica y al compromiso a nivel ventilatorio, se consideró iniciar la ventilación de alta frecuencia, sin obtener buena respuesta clínica, dada la persistencia de hipoxemia y de episodios de bradicardia sostenida.

En su tercer día de vida, el recién nacido presentó un nuevo episodio de bradicardia grave. Se inició la reanimación neonatal y el manejo farmacológico con adrenalina por alrededor de 20 minutos, sin la recuperación de su estado hemodinámico. El paciente falleció, pese a los esfuerzos del personal médico. Se obtuvo el

TABLA 1. Exámenes de laboratorio en las primeras 6 horas de vida

\begin{tabular}{lcc}
\hline Laboratorio & Resultado & Valor de referencia \\
\hline Leucocitos & 7740 & $9000-30000 \mathrm{~mm}^{3}$ \\
Neutrófilos (\%) & $64 \%$ & $30-75 \%$ \\
Neutrófilos (absolutos) & 4954 & $6000-26000 \mathrm{~mm}^{3}$ \\
Linfocitos (\%) & $22 \%$ & $24-41 \%$ \\
Linfocitos (absolutos) & 1702 & $2000-11000 \mathrm{~mm}^{3}$ \\
Eosinófilos (\%) & $2 \%$ & $0-2 \%$ \\
Eosinófilos (absolutos) & 155 & $0-400 \mathrm{~mm}^{3}$ \\
Hemoglobina & $18,1 \mathrm{~g} / \mathrm{dl}$ & $13-19 \mathrm{~g} / \mathrm{dl}$ \\
Hematocrito & $55,8 \%$ & $47-59 \%$ \\
Plaquetas & $136500 / \mathrm{mm}$ & $180000-327000 \mathrm{~mm}^{3}$ \\
Calcio & $8,5 \mathrm{mg} / \mathrm{dl}$ & $7,6-10,4 \mathrm{mg} / \mathrm{dl}$ \\
Cloro & $107 \mathrm{mmol} / \mathrm{l}$ & $98-107 \mathrm{mmol} / 1$ \\
Fósforo & $3,7 \mathrm{mg} / \mathrm{dl}$ & $3,9-7,7 \mathrm{mg} / \mathrm{dl}$ \\
Magnesio & $3,6 \mathrm{mg} / \mathrm{dl}$ & $1,7-2,5 \mathrm{mg} / \mathrm{dl}$ \\
Potasio & $4,06 \mathrm{mmol} / \mathrm{l}$ & $3,5-5,3 \mathrm{mmol} / 1$ \\
Sodio & $141 \mathrm{mmol} / \mathrm{l}$ & $135-145 \mathrm{mmol} / 1$ \\
PCR & $11 \mathrm{mg} / \mathrm{l}$ & $<5 \mathrm{mg} / 1$ \\
Bilirrubina total & $5,73 \mathrm{mg} / \mathrm{dl}$ & $0,3-1 \mathrm{mg} / \mathrm{dl}$ \\
Bilirrubina directa & $1,64 \mathrm{mg} / \mathrm{dl}$ & $0-1 \mathrm{mg} / \mathrm{dl}$ \\
Bilirrubina indirecta & $4,09 \mathrm{mg} / \mathrm{dl}$ & $0,6-10,5 \mathrm{mg} / \mathrm{dl}$ \\
Hemocultivo \# 1: ${ }^{*}$ positivo a las 30 horas de incubación para Lactobacillus reuteri &
\end{tabular}

Hemocultivo \# 2:* positivo a las 32 horas de incubación para Lactobacillus reuteri

* Confirmados de forma institucional y extrainstitucional, dada la etiología atípica evidenciada.

PCR: proteína C reactiva. 
reporte oficial de hemocultivos con crecimiento de Lactobacillus reuteri, que fue confirmado a nivel institucional y extrainstitucional por sus características microbiológicas típicas.

\section{DISCUSIÓN}

La sepsis neonatal continúa siendo una entidad frecuente y de gran impacto en la mortalidad y morbilidad en este grupo etario. ${ }^{1,2}$ La utilización de probióticos en la población infantil y neonatal, como el Lactobacillus reuteri, una bacteria Grampositiva anaerobia aerotolerante, ha aumentado significativamente en los últimos años debido a los múltiples efectos benéficos descritos. ${ }^{3}$

Los probióticos son microorganismos que, administrados en dosis adecuadas, brindan efectos favorables al huésped. ${ }^{4}$ Se ha documentado que la utilización rutinaria de probióticos en la población neonatal mejora el funcionamiento de la microbiota intestinal; promueve la madurez y la función de la barrera intestinal;, ${ }^{5,6}$ disminuye la pérdida de bacterias comensales, tales como
Bifidobacterium y Lactobacillus; reduce el tiempo para el inicio de la alimentación enteral plena; disminuye los episodios de intolerancia a la vía oral $^{5,6}$ y mejora la ganancia de peso.

Adicionalmente, revisiones sistemáticas confirman que el suplemento con probióticos disminuye significativamente la mortalidad y la incidencia de entidades como enterocolitis necrosante y sus complicaciones (sepsis recurrente, necesidad de múltiples intervenciones, síndrome de intestino corto y alteraciones en el neurodesarrollo), principalmente, en RNPT, ${ }^{2,7}$

Además, estudios en animales destacan la capacidad que posee el Lactobacillus reuteri para disminuir la expresión de citoquinas proinflamatorias y favorecer la liberación de citoquinas antiinflamatorias a través de su efecto sobre células T. ${ }^{2}$ A su vez, los Lactobacillus, a través del proceso de fermentación láctica, favorecen la producción de sustancias antimicrobianas (ácido acético, ácido láctico, peróxido de hidrógeno). ${ }^{8}$

Se presenta un caso atípico de sepsis neonatal

TABLA 2. Reportes de casos de sepsis por probióticos en neonatos y niños

\begin{tabular}{|c|c|c|c|}
\hline & Edad & Factores de riesgo & Etiología \\
\hline Kunz et al. ${ }^{8}$ & $\begin{array}{l}3 \text { meses } \\
10 \text { sem. }\end{array}$ & $\begin{array}{l}\text { Prematuridad, SIC } \\
\text { Prematuridad, gastrosquisis, SIC }\end{array}$ & Lactobacillus rhamnosus \\
\hline De Groote et al. ${ }^{1}$ & 11 meses & Prematuridad, gastrosquisis, SIC, CVC & Lactobacillus rhamnosus \\
\hline Land et al. ${ }^{1}$ & $\begin{array}{l}6 \text { sem. } \\
6 \text { años }\end{array}$ & $\begin{array}{l}\text { CC, diarrea relacionada con antibióticos } \\
\text { Parálisis cerebral, yeyunostomía, CVC, } \\
\text { diarrea relacionada con antibióticos }\end{array}$ & Lactobacillus rhamnosus \\
\hline Sadowska-Krawczenko et al. ${ }^{4}$ & 6 días & RCIU & Lactobacillus rhamnosus \\
\hline Carlo Dani et al. ${ }^{1}$ & $\begin{array}{l}3 \text { meses } \\
18 \text { días }\end{array}$ & $\begin{array}{c}\text { Trisomía 18, síndrome X triple, CC, CVC } \\
\text { Prematuridad, DAP, CVC }\end{array}$ & Lactobacillus rhamnosus \\
\hline Esaiassen et al. ${ }^{12}$ & $\begin{array}{l}2 \text { sem. } \\
2 \text { sem. } \\
3 \text { sem. } \\
5 \text { sem. }\end{array}$ & $\begin{array}{l}\text { Prematuridad } \\
\text { Prematuridad } \\
\text { Prematuridad } \\
\text { Prematuridad }\end{array}$ & Bifidobacterium longum \\
\hline Ohishi A. et al. ${ }^{3}$ & 6 sem. & VDDS & Bifidobacterium breve \\
\hline Jenke et al. ${ }^{3}$ & 18 días & Prematuridad, STFF & Bifidobacterium spp. (longum e infantis) \\
\hline Zbinden et al. $^{7}$ & $\begin{array}{l}20 \text { días } \\
20 \text { días } \\
11 \text { días }\end{array}$ & $\begin{array}{c}\text { Prematuridad } \\
\text { Prematuridad } \\
\text { Prematuridad, EN (estadio III) }\end{array}$ & Bifidobacterium longum \\
\hline Bertelli et al. ${ }^{3}$ & $\begin{array}{l}10 \text { días } \\
14 \text { días }\end{array}$ & $\begin{array}{l}\text { Prematuridad, EN (estadio III) } \\
\text { Prematuridad }\end{array}$ & Bifidobacterium longum \\
\hline Brecht et al. ${ }^{3}$ & 63 días & Prematuridad & Lactobacillus rhamnosus \\
\hline Roy et al. ${ }^{13}$ & $\begin{array}{l}\text { Neonato } \\
\text { Neonato }\end{array}$ & $\begin{array}{c}\text { Prematuridad } \\
\text { Prematuridad, CVC }\end{array}$ & Saccharomyces boulardii \\
\hline Celis et al. (caso actual) & 2 días & Prematuridad & Lactobacillus reuteri \\
\hline
\end{tabular}

CC: cardiopatía congénita; CVC: catéter venoso central; RCIU: restricción del crecimiento intrauterino;

DAP: ductus arterioso persistente; VDDS: ventrículo derecho de doble salida; EN: enterocolitis necrosante;

SIC: síndrome de intestino corto; STFF: síndrome de transfusión feto-fetal. 
en un RNPT por Lactobacillus reuteri, el cual constituye el primer caso reportado en nuestra Unidad Neonatal luego de más de 10 años de utilización de dicho probiótico. Se observa a un RNPT extremo sin evidencia de infección intrauterina, que desarrolla, durante su estancia temprana, distermias, múltiples episodios de apneas y deterioro progresivo en su estado hemodinámico y respiratorio compatible con sepsis neonatal.

Las manifestaciones clínicas de bacteriemia por Lactobacillus son amplias y pueden variar desde un estado asintomático hasta un cuadro de sepsis grave (este último, observado en nuestro paciente). ${ }^{9}$ Otras manifestaciones menos frecuentes son neumonía, abscesos abdominales y endocarditis. Generalmente, se documenta, además, la presencia de fiebre, proteína $C$ reactiva (PCR) elevada y leucocitosis franca. ${ }^{9} \mathrm{En}$ nuestro paciente, en particular, no se evidencia el desarrollo de leucocitosis, que puede estar asociado a su condición de extrema inmadurez.

Pese a los efectos favorables descritos, se debe tener precaución en la administración de probióticos en los pacientes de alto riesgo, tales como inmunosuprimidos, usuarios de catéteres endovenosos, con síndrome de malabsorción, con cirugía previa y con esquemas de antibioticoterapia prolongados, ${ }^{4}$ debido a que se han descrito reportes de casos de sepsis neonatal por Lactobacillus rhamnosus, Saccharomyces boulardii y Bifidobacterium, entre otros (Tabla 2). ${ }^{3}$ Los RNPT poseen un mayor riesgo de bacteriemia y/o fungemia por probióticos debido a la inmadurez de su sistema inmune. ${ }^{10}$

En la actualidad, no existen pautas o criterios de selección específicos para el suplemento con probióticos en la población infantil o neonatal. Algunos autores sugieren evitar el Lactobacillus reuteri en los recién nacidos con peso $\leq 1800$ gramos o restricción del crecimiento intrauterino, puesto que no existen datos epidemiológicos convincentes. ${ }^{4}$ Sin embargo, otros estudios destacan la reducción de enterocolitis necrosante y sepsis tardía en $<1000$ gramos luego del suplemento con dicho probiótico, ${ }^{2}$ por lo que continúan siendo necesarios estudios encargados de documentar la seguridad y eficacia de esta medicación.

Se destaca, además, la especie en particular de Lactobacillus aislado en nuestro caso clínico (Lactobacillus reuteri), puesto que las dos especies más frecuentemente reportadas como responsables de sepsis o bacteriemia son
Lactobacillus casei y Lactobacillus rhamnosus. ${ }^{9}$ Los hemocultivos, en nuestro caso clínico, fueron procesados tanto a nivel institucional como extrainstitucional, debido al aislamiento de un microorganismo poco evidenciado en la población pediátrica y neonatal.

Históricamente, nuestra Institución ha manejado a un grupo poblacional de alto riesgo, dadas las limitadas condiciones socioeconómicas de los pacientes atendidos. En 1999, en nuestro Servicio, A. B. Hoyos encontró una reducción en la incidencia de enterocolitis necrosante y de mortalidad en los pacientes prematuros que habían recibido probióticos. ${ }^{11}$ Sin embargo, debido a la poca disponibilidad del medicamento y a la no cobertura por parte de nuestro sistema de salud, dicha intervención fue interrumpida alrededor del año 2000 y se observó un incremento de las tasas de enterocolitis necrosante y sepsis neonatal, que oscilaban entre un 10-30\%.

Para contrarrestar esta situación y con el objetivo de disminuir la incidencia de estas patologías, se implementaron y ajustaron, en nuestra Unidad, las estrategias recomendadas por la literatura, como el inicio más temprano de la vía enteral mediante el estímulo trófico, el uso de leche materna y el control de antibióticos. Sin embargo, debido a la limitación en la disponibilidad de leche materna por las características sociodemográficas de la población, se consideró, en conjunto con el Comité de Infecciones, la implementación de la administración de probióticos (Lactobacillus reuteri) a todos los recién nacidos hospitalizados en nuestra Institución, y se logró una reducción anual, en promedio, del $8 \%$ de los casos de enterocolitis necrosante y del $15 \%$ en sepsis neonatal temprana a partir del año 2003.

Se debe recordar la importancia de realizar una adecuada selección del paciente candidato al inicio del suplemento con probióticos para reducir los efectos desfavorables e identificar a aquellos que, por su estado clínico o hemodinámico, poseen un epitelio intestinal disfuncional o inmaduro, que puede incrementar el riesgo de complicaciones $y$, sin duda, afecta la supervivencia del paciente, tal y como lo describe nuestro caso.

\section{Agradecimientos}

Los autores agradecen a la Unidad de Neonatología del Hospital Simón Bolívar (HSB) por la asesoría académica prestada durante la realización de este trabajo. 


\section{REFERENCIAS}

1. Dani C, Coviello C, Corsini I, Arena F, et al. Lactobacillus Sepsis and Probiotic Therapy in Newborns: Two New Cases and Literature Review. AJP Rep. 2016; 6(1):e25-9.

2. Athalye-Jape G, Rao S, Patole S. Lactobacillus reuteri DSM 17938 as a Probiotic for Preterm Neonates: A Strain-Specific Systematic Review. JPEN J Parenter Enteral Nutr. 2016; 40(6):783-94.

3. Brecht M, Garg A, Longstaff K, Cooper C, Andersen C. Lactobacillus Sepsis following a Laparotomy in a Preterm Infant: A Note of Caution. Neonatology. 2016; 109(3): 1869.

4. Sadowska-Krawczenko I, Paprzycka M, Korbal P, Wiatrzyk A, et al. Lactobacillus rhamnosus GG suspected infection in a newborn with intrauterine growth restriction. Benef Microbes. 2014; 5(4):397-402.

5. Van den Akker CHP, Van Goudoever JB, Szajewska H, Embleton ND, et al. Probiotics for Preterm Infants: A StrainSpecific Systematic Review and Network Meta-analysis. J Pediatr Gastroenterol Nutr. 2018; 67(1):103-22.

6. Manzoni P. Use of Lactobacillus casei subspecies Rhamnosus GG and gastrointestinal colonization by Candida species in preterm neonates. J Pediatr Gastroenterol Nutr. 2007; 45(Suppl 3):S190-4.
7. Zbinden A, Zbinden R, Berger C, Arlettaz R. Case series of Bifidobacterium longum bacteremia in three preterm infants on probiotic therapy. Neonatology. 2015; 107(1):56-9.

8. Kunz AN, NoelJM, FairchokMP. Two cases of Lactobacillus bacteremia during probiotic treatment of short gut syndrome. J Pediatr Gastroenterol Nutr. 2004; 38(4):457-8.

9. Salminen MK, Rautelin H, Tynkkynen S, Poussa T, et al. Lactobacillus bacteremia, clinical significance, and patient outcome, with special focus on probiotic L. rhamnosus GG. Clin Infect Dis. 2004; 38(1):62-9.

10. Thompson C, McCarter YS, Krause PJ, Herson VC. Lactobacillus acidophilus sepsis in a neonate. J Perinatol.2001; 21(4):258-60.

11. Hoyos AB. Reduced incidence of necrotizing enterocolitis associated with enteral administration of Lactobacillus acidophilus and Bifidobacterium infantis to neonates in an intensive care unit. Int J Infect Dis. 1999; 3(4):197-202.

12. EsaiassenE,HjerdeE,CavanaghJP,SimonsenGS;Norwegian Study Group on Invasive Bifidobacterial Infections. Bifidobacterium Bacteremia: Clinical Characteristics and a Genomic Approach To Assess Pathogenicity. J Clin Microbiol. 2017; 55(7):2234-48.

13. Roy U, Jessani LG, Rudramurthy SM, Gopalakrishnan R, et al. Seven cases of Saccharomyces fungaemia related to use of probiotics. Mycoses. 2017; 60(6):375-80. 